\title{
Design and Realization of a Handheld Terminal of Consumption Data Acquisition
}

\author{
Chuanfei Qiu, Quanzhi Zhou, Jingjing Zhou, Xiaofang Lou and Weiqing Tian \\ Ordnance NCO Academy, Arm Engineering University of PLA, Wuhan Hubei 430075, China
}

\begin{abstract}
Consumption data not only constitute the basic premise for analysis of, but also provide the basic reference for production, ordering, storage and supply of maintenance materials. This article designs a handheld terminal of consumption data acquisition, which can greatly overcome the disadvantages of low efficiency and incomplete data of the original acquisition method.
\end{abstract}

Keywords-data acquisition; data synchronization; management analysis; data backup

\section{INTRODUCTION}

The system consumption data are the basic premise for analysis of consumption, and also the basic reference for production, ordering, storage and supply of maintenance materials. However, there are no advanced and efficient consumption data acquisition technologies, and the efficiency and management of manual data input into tables are subject to severe constraints and restrictions.

Due to the rich variety of maintenance materials and different amounts of consumption, a mass of data need to be collected and managed. Currently three methods are mainly utilized for acquisition of the system consumption data. First, the data are manually collected and reported in tables for summary; second, equipment manufacturers provide statistics of maintenance material production and equipment repair records; third, the equipment management unit collects purchase data of maintenance materials. Finally, the system consumption data are comprehensively formed.

There are four disadvantages for the current acquisition of system consumption data. First, low efficiency by reason of time-consuming data acquisition from many users; second, incomplete data acquisition because many users do not or have no full time employees allocated for this task and data collected by temporary staff may be incorrect; third, repetition in data acquisition when materials are delivered from the manufacturer, distributed to the management unit and allocated to users; fourth, difficulty in data processing because manually collected consumption data are not standardized and unified.

These four disadvantages finally result in inaccurate analysis of consumption of maintenance materials and inability to complete production, ordering, storage and supply pursuant to the actual consumption. Thus deficiencies in material consumption cannot be made up or equipment fault cannot be repaired promptly, and some materials are idle for long and lead to a waste of resources.
Considering the aforesaid four disadvantages, it is impossible for researchers and suppliers to obtain accurate data for analysis of material consumption and reasonably and scientifically make arrangements for production, ordering, storage and supply of maintenance materials. Therefore, the system consumption data acquisition device can perfectly overcome these disadvantages and solve relevant problems.

\section{SYSTEM FUNCTION DESIGN}

Acquisition of 1D and 2D barcode data by the handheld terminal enables the management of local database, centralized processing of device information and synchronized management of local database and host database.

\section{A. Data Management Function}

The data management function mainly involves provision of material barcode information for acquisition and manual input; record of material stock-in/out information; statistics of material stock and consumption data; bulk import and export of data; and also account management function to enhance data confidentiality.

\section{B. Data Query and Display Function}

The data query and display function supports query and display of data of material stock and consumption; and query and display of related circuit diagrams, which can be zoomed in or out.

\section{Data Synchronization Function}

The data synchronization function supports regular data backup; completes data transmission, update and synchronization between host and terminal device; and exports data and supports the extension network reporting function.

\section{SYSTEM STRUCTURE DESIGN}

\section{A. System Solution}

Firstly, maintenance materials are coded with $1 \mathrm{D}$ and 2D barcodes, which affixed to package. While replacing parts, maintenance staff of manufacturer or user may scan these barcodes to collect consumption data with the data acquisition device. The collected standard and unified material consumption data are then exported for summary and processing by utilizing the data management and automatic backup function. This can thoroughly overcome disadvantages and deficiencies of the current technologies. 


\section{B. System Composition}

The system consumption data acquisition terminal adopts the ARM technology system, uses the ARM-Cortex A8 CPU core system as the hardware platform and transplants Wince6.0 embedded operating system as the software platform. Based on the design of the human-computer interaction (HCI) software, the barcode are decoded for reading. The graphics support module is developed for product association with the barcode data acquisition. Then the data management and automatic backup module is developed and match with the designed communication interface for achieving data management and automatic backup function. The power management module is used for power charging and supply management. The miniaturization design is conducted so that the data acquisition terminal is miniaturized and portable. Various functions of the terminal can be realized on this basis. Therefore, this terminal comprises the hardware and software systems. The hardware system is made up of core system, touch screen, camera, communication interface and power management module, and the software system is composed of operating system, equipment drive, database and HCI module. The structure is presented in Figure I as follows.

\begin{tabular}{|c|c|c|c|c|c|}
\hline $\mathrm{HCI}$ & $\begin{array}{l}\text { Graphics } \\
\text { support } \\
\text { module }\end{array}$ & \multicolumn{2}{|c|}{\begin{tabular}{|c|} 
Barcode \\
reading/decode \\
module
\end{tabular}} & \multicolumn{2}{|c|}{$\begin{array}{c}\text { Data } \\
\text { management \& } \\
\text { automatic backup }\end{array}$} \\
\hline \multicolumn{6}{|c|}{ Database } \\
\hline \multicolumn{6}{|c|}{ Equipment drive } \\
\hline \multicolumn{6}{|c|}{ Wince6.0 embedded operating system } \\
\hline \multicolumn{6}{|c|}{ ARM-Cortex $A 8$ core system } \\
\hline $\begin{array}{l}\text { Touch } \\
\text { screen }\end{array}$ & Keypad & Camera & $\begin{array}{l}\text { Commun } \\
\text {-on inter }\end{array}$ & $\begin{array}{l}\text { icati } \\
\text { face }\end{array}$ & $\begin{array}{c}\text { Power } \\
\text { management } \\
\text { module }\end{array}$ \\
\hline
\end{tabular}

FIGURE I. SYSTEM CONSUMPTION DATA ACQUISITION EQUIPMENT ARCHITECTURE

\section{Module Design}

\section{A. Core System Design}

An ARM-Cortex A8 CPU is used as the control core of the core system. It has the single-core clock speed of $1 \mathrm{GHz}$ and the extension of 4GB ROM and 256M RAM [1], which can support smooth running of Wince6.0 operating system. The industrial grade Cortex A8 can be stable and reliable with abundant resources, thus being easy for system extension. ARM-Cortex A8 CPU mini system and peripheral circuits constitute part of hardware modules, such as communication bus, LCD, barcode reader module, database management, power module and SD card management. There are also abundant redundancy of hardware resources, such as IO resource and communication port, which allows for future system extension. The composition diagram is presented in Figure II.

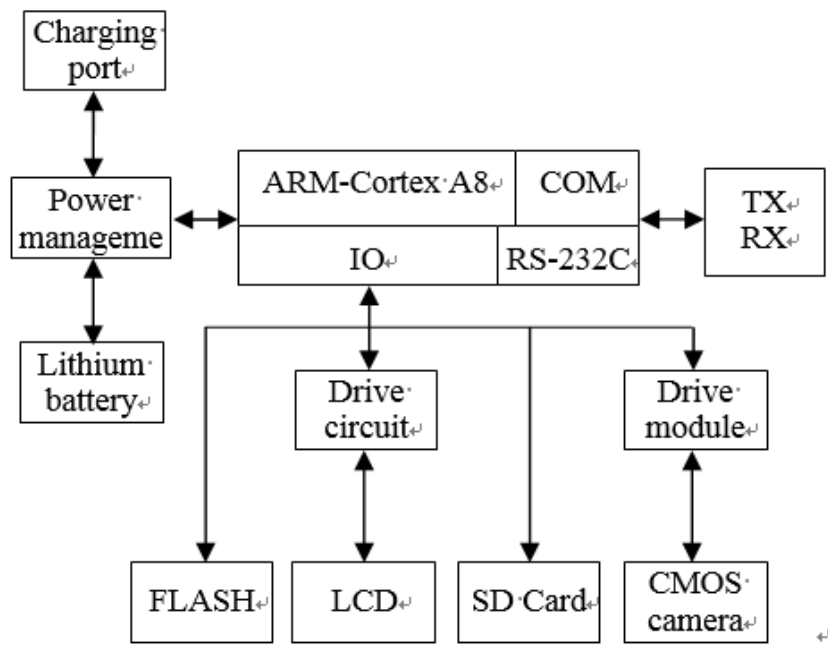

FIGURE II. CORE SYSTEM STRUCTURE

\section{B. Transplant of Operating System}

The system consumption data acquisition terminal uses the embedded operating system Wince6.0, which can meet the system performance requirements [2].

Windows CE is the basis for the Microsoft embedded and mobile computer platforms and an open and upgradable 32-bit embedded system. It is an OS for electronic devices based on pocket PCs and its graphical user interface is very excellent. The system is transplanted to our terminal.

Windows CE is a multi-task operating system supporting priority levels and allowing for multiple functions and processes. Windows CE running in the same time system can support the maximum of 32-bit synchronous processes. A process may contain one or more threads and each thread is a separate part of a process. A thread can be designated as a basic thread of a process, and a process can create an undetermined number additional threads. The number of additional threads can only be restricted by the available system resources.

On the embedded hardware platform, this terminal transplants Wince6.0 operating system as the software support platform and integrates the user layer applications, such as camera drive, decoding, barcode scanning and data management.

\section{Communication Interface Design}

This terminal provides a USB port for data transmission. In order to ensure the security of data transmission, it does not provide any wireless or wired connections.

The standard USB2.0 port is used as the communication interface driven by the Windows CE specific drive software ActiveSync6 [3]. It can achieve wireless connection of handheld terminal and PC management software and further realize advanced management such as data synchronization with the data management system. 


\section{Barcode Decoding Design}

This terminal supports decoding of $1 \mathrm{D}$ and 2D barcodes. At present, the mainstream scanning and decoding systems utilize CMOS cameras to capture 1D and 2D barcodes. The software runs decoding to obtain data information. Common decoding modules can simultaneously support decoding of $1 \mathrm{D}$ and $2 \mathrm{D}$ barcodes.

$1 \mathrm{D}$ and 2D barcodes have a variety of encoding formats or types. This device imposes certain restrictions on the types of barcodes (basically including commonly used formats), that is, the encoding of actual devices must be within the scope of this specification to ensure that the system can recognize them normally.

This device supports these 1D barcode formats: Code 39,Interleaved 2 of 5, Codabar, Full ASCII Code 39, Code 128, Industrial 2 of 5, Code11,Code93, UCC/EAN128, UPC-A, UPC-E, EAN13, EAN8,MSI. [4]

This device supports these 2D barcode formats: PDF417, QR Code (Model 1/2), DataMatrix (ECC200, ECC000, 050, 080, 100, 140), Aztec, Maxicode.

\section{E. Graphics Support Design}

This device supports photo shooting and basic management functions.

With an independent 500W pixel camera on the back of the device, it can take the required picture files, store them and freely associate them to the product.

Pictures support basic management operations such as browsing, association, disassociation and deletion. The pictures may be automatically synchronized when connected to a PC data management system.

\section{F. HCI Design}

The device needs to implement barcode data identification (database query), entry (new device information), and operation (such as stock-in/out). Therefore, the HCI interface of this device includes display, information input and other designs.

HCI interface is friendly in design and convenient for operation, including the core functions of entry information query and modification, operation record query and editing, picture management, system configuration, automatic backup, and data synchronization. The main interface design of HCI software is shown in Figure III.

The device realizes the HCI interface design based on a touchable color LCD for display of graphics and texts, information input and other operations. Taking account of size, power consumption and ease of operation, a 3.2-inch touchable color dot matrix LCD screen is used.

The device also has a 33-key physical keyboard, which is easy for operation and has the basic functions such as scanning trigger, power, reset and number input. As a supplement to the touch screen, the physical keyboard can effectively improve the ease of use of the handheld terminal.

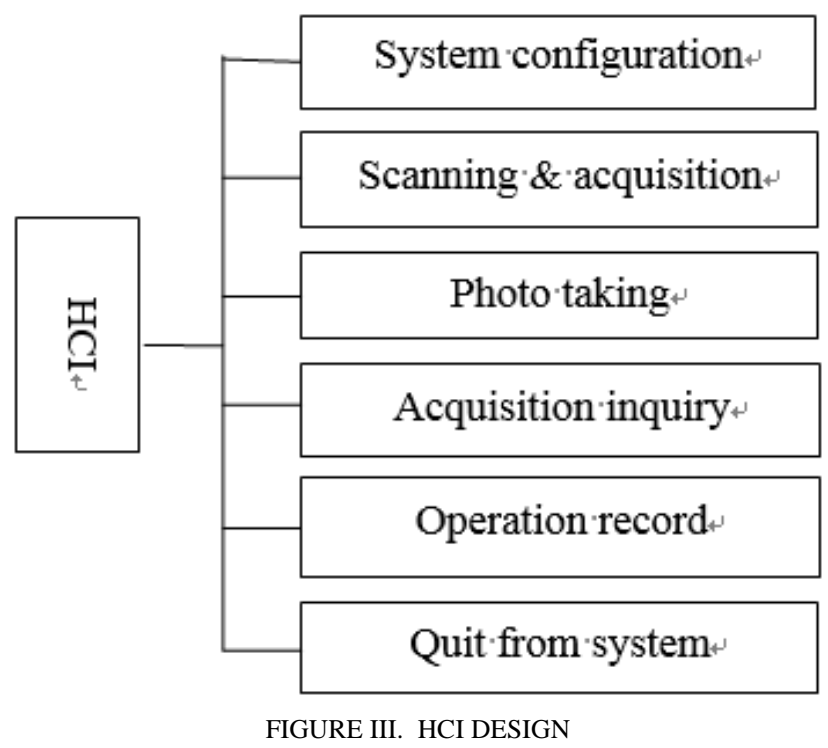

G. Data Management and Automatic Backup Design

This system consumption data acquisition device has a local database designed with basic operations such as query, addition and modification. Given the process capability and actual need for use of the core system, the streamlined data structure management is adopted. The SD card is used for the storage of automatic backup data, and it can support a maximum of 32G SD card. The core advantage of choosing an SD card as the data storage carrier is pluggable so that it is easy for the synchronization of computer management software and device database and the management of data files, and ensures that data files are not lost even if the device does not work properly.

The basic management operations of the database are realized with the management software, which must take account of the consistency and compatibility with the database content of the computer management software, so as to facilitate synchronous management. The formats of data storage need to be specially designed for the hardware environment of the device. The data management diagram is shown in Figure IV.

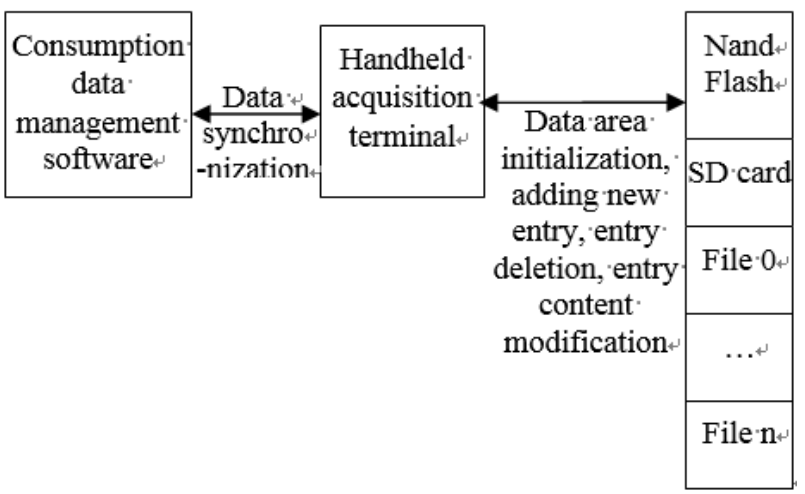

FIGURE IV. DATABASE MANAGEMENT DESIGN 
The automatic data backup adopts the user trigger mode. When the user completes the related operations of the data acquisition device and manually quits the application software, the program automatically backs up the current key files to the SD card backup directory, such as product entries and operation records, thereby ensuring the data reliability.

If the acquisition device is unable to acquire data normally due to accidental data destruction or failure to boot as a result of system crash, the historical key data can be obtained from the SD card, which enhances the reliability of data storage.

\section{H. Power Management Design}

The data acquisition device is powered by a removable rechargeable lithium battery, whose advantages include sufficient power, recharging, good structural sealing and long service life. In combination with the actual application scenario of the device, a high-capacity lithium battery is selected as the power supply for the data acquisition device. The theoretical standby time is not less than 100 hours, so as to meet the conditions for actual use.

A dedicated power management module is designed for managing the charge and discharge of the lithium battery, ensuring stable and reliable operation of the power source and prolonging the service life of lithium battery as much as possible.

Meanwhile, the software sets an automatic standby mode to reduce the power consumption and increase the continuous working time of the device.

\section{CONCLUSIONS}

Through unified coding, the maintenance materials of the system have standard and unified names, which are convenient for unified processing and analysis. Maintenance staff undertake the operations of scanning, acquisition and input by using the system consumption data acquisition device. These operations are simple and quick, overcome the trouble and solve the problems of unwillingness or omission in data acquisition. Also, it solves the problem of repeated acquisition of consumption data because maintenance staffs collect data only during replacement of parts and repair. The device can achieve management and automatic backup of collected data and export the data for summary and processing. This solves not only the difficulty in summarizing consumption data, but also the problem of data processing. These are the great benefits brought by the system consumption data acquisition device.

Moreover, the system consumption data acquisition device can also be applied to other types of system equipment or other types of equipment consumption data acquisition for solving the problems of other equipment.

\section{REFERENCES}

[1] Wang Heng, In-depth analysis of ARM Cortex-A8. Biejing: Electronics Industry Press, 2016, pp. 6-8.

[2] Feng Zhangdongsong, Chen Fangguo, Windows CE 6 embedded advanced programming. Biejing: Tsinghua University Press, 2009, pp. $10-11$
[3] Patrick Alessi, Mobile development classic series. Biejing: Tsinghua University Press, 2013, pp. 176-179.

[4] Standardization Administration of the People's Republic of China, SAC, General technical specification for two-dimensional code-GB/T 310222014. Biejing: Standards Press of China, 2014, pp.15-16 